\title{
Functional Modeling of Monitoring \& Diagnosis System Based on Multi-Agents
}

\author{
(Dept. of 5, Wuhan Mechanical Technology, Wuhan 430075, China) \\ Email:1712178221@qq.com.cn
}

\begin{abstract}
Intelligent monitoring and diagnosis is the development trend of diagnosing failures in modern times. This article contrives a method for functional modeling of monitoring and diagnosis system based on multi-agents in the conception of modularization, and expounds the internal configuration of each functional Agent in the model, which is featured by uniform information interfaces, and construction of the system with different functional components depending upon various objects to be monitored. The model is valuable to guide realizing intelligent monitoring and diagnosis system, and helpful in constructing integrated, intelligent, automated and networked diagnosis system.

Key words-Agent; Monitoring and Diagnosis; Function
\end{abstract} Model

\section{Foreword}

Complex equipment is highly integrated with increasingly complicated configuration, leading to frequent unexpected and coincident failures in the process of system operation. Moreover, due to variable monitoring environments, it's impossible to predetermine an optimal diagnostic method or procedure even for the same equipment; however, human expert usually tends to select an appropriate diagnostic method or procedure that is unable to change with the environment, thus degrading efficiency and level of automation as well as resultant lower adaptability of diagnosis system.

The monitoring and diagnosis system based on multi-Agents, characterized by agent, intelligence and mobility, is essentially adapted to the requirements of real-time and coincident processing in the development of engineering applications. By working with Agent, knowledge, information and information resources about spatial distribution are fully utilized to strengthen adaptability of monitoring and diagnosis system to dynamic environment, and enhance its capacity in processing incomplete information, so as to realize distributed computing and solutions for problems in network environment.

II Function Model of Monitoring \& Diagnosis System Based on Multi-Agents

Generally, in order to construct a multi-Agents diagnosis system, the first thing to do is formal description of diagnosis tasks or targets as well as subdivision and decomposition of tasks, for proper and logic decomposition of tasks enables use of less diagnosis resources with higher real-time efficiency and diagnosis accuracy of diagnosis system. Role-based decomposition of mixed tasks is adopted in the construction of the model, which integrates multiple decomposition of tasks such as hierarchy decomposition and diagnosis method decomposition, and allies object modeling and role modeling, for decomposition of diagnosis tasks by the merits of all these methods, with following advantages: (1) decomposition based on failure diagnosis process, by which solution field of Agent will not superpose by avoiding redundant reasoning, so as to increase diagnosis efficiency; (2) description of role model embodies coordination among Agents; and (3) decomposition granularity is moderate, which helps construction of Agent-based diagnosis system. Furthermore, the conception of modularization is represented in realizing each role, in that each module corresponds to an information interface, information communication among Agents is realized for coordinated accomplishment of diagnosis task according to the requirement of the system for diagnosis activity, for different diagnosis objects or tasks, different functional Agents may have different functions, and modification of corresponding functional Agent will work, thus this function model substantially enhances re-configurability and flexibility of the system, leading to powerful system openness and adaptability. The model consists mainly of the following functional modules: monitoring Agent, feature extraction Agent, failure diagnosis Agent, forecast Agent, decision-making Agent, interfacing Agent and administration Agent.

\section{A. Monitoring Agent}

Monitoring Agent is the main source of information in monitoring and diagnosis system based on multi-Agents, as well as basic expression of perception ability of Agent, and which is equivalent to reactive Agent. Monitoring Agent is mainly composed of modules including intelligent sensor, signal filter, state analysis and list of standard signal states, and its configuration is illustrated in Figure 1. Monitoring Agent functions to choose different intelligent sensor for real-time monitoring according to signal type of the object to be monitored, smooth filtering of monitored data for obtaining information on actual data, following by state analysis and comparison with list of standard signal states, so as to judge current operating state of the equipment, if abnormal state data is discovered, diagnosis request is sent to diagnosis Agent or to featured extraction Agent for extraction of state feature ready for final failure diagnosis.

\section{B. Feature Extraction Agent}

For large complex electronic device, which may monitor dozens and even hundreds of points on a real-time basis, tremendous time and computing resources will be consumed for thorough and comprehensive analysis of monitored data in the event of failure of such electronic device in operation; on the other hand, equal or similar tendency of variation may exist with the same parameter in different modes of failure, leading to difficulty in the process of failure analysis, and 
accurate judgment of the cause and location of failure. Therefore, it's very important for extraction of failure feature of complex system in the whole process of failure diagnosis, in terms of immediate impact on computing load of whole diagnosis process and performance of diagnosis system.

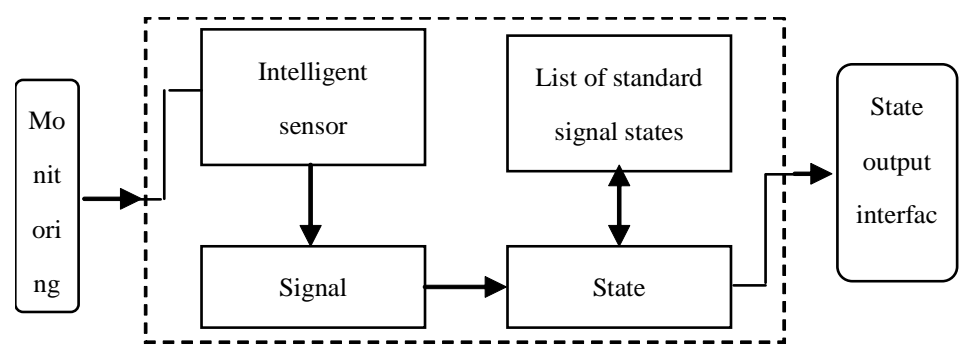

Fig. 1 Functional Configuration of Monitoring Agent

Primary principle of Feature extraction Agent is that, high-dimensional space of original failure features is expressed by new pattern vectors of low-dimensional space of original failure features by means of transformation (Or mapping), so as to find most representative and effective failure features. Feature extraction Agent functions for feature extraction by selecting different feature extraction method for organizing state data in terms of the type and feature of state data, which is helpful for failure diagnosis, and its configuration is illustrated in Figure 2.

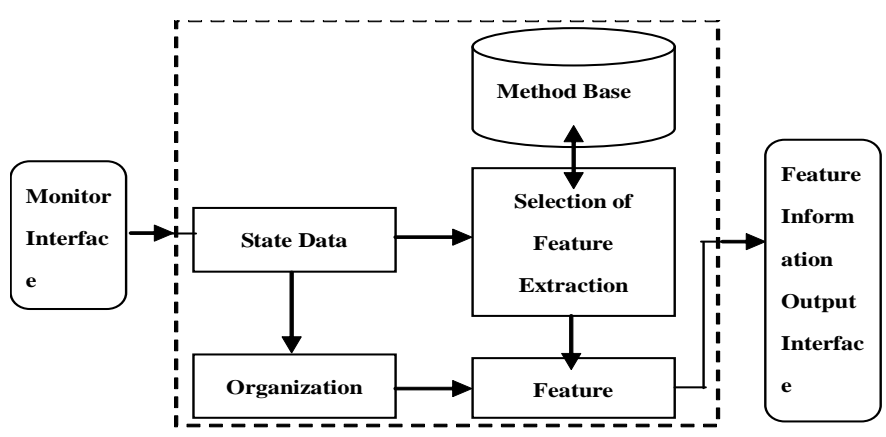

Fig. 2 Functional Configuration of Feature Extraction Agent

\section{Failure Diagnosis Agent}

Failure diagnosis Agent functions for diagnosis and explanation of abnormality and failure of the monitoring object, so as to determine the cause and location of failure. Diagnosis information is mainly from monitoring Agent or feature extraction Agent, and its configuration is illustrated in Figure 3. The model mainly consists of shallow and deep knowledge reasoning mechanisms, between which there is a public data area of blackboard structure for storage of failure phenomenon, failure hypothesis and failure conclusions for sharing data. In the process of reasoning, shallow knowledge reasoning mechanism is first activated, and failure conclusion will be made with explanation and ending of reasoning process if failure cause is received successfully, or else some most possible failure hypothesis will be generated before reaffirmation of such hypothesis by deep knowledge reasoning mechanism, and finally make conclusion on such failure. Particularly, failure diagnosis Agent has following main functions:

(1) Upon discovery of abnormal phenomenon by monitoring Agent, it'll automatically execute failure diagnosis, usually diagnosis by means of artificial intelligence, such as expert system diagnosis method and case-based diagnosis method, all of which fall into the category of shallow knowledge diagnosis.

( 2 ) In-depth failure diagnosis by integrating the information on various signs. Knowledge used for deep diagnosis mainly includes: (1) actually monitored data, including currently measured data and history data; (2) Structural knowledge, mainly of analytical data from failure mode effects and criticality analysis (FMECA), by which analysis starts with the effect and corresponding criticality of inferior component to superior component, failure reasoning is carried out by criticality to realize qualitative and quantitative diagnosis; (3) failure record knowledge is used to record frequent failures of equipment; and (4) knowledge about failure maintenance records is used to reflect frequency of equipment failure, so as to provide history evidence for failure diagnosis.

(3) Different diagnosis methods can be selected by requirements, and each diagnosis method is deemed as one diagnosis Agent for coordinated diagnosis; Different diagnosis method may produce result of different and even contrary confidence, thus evaluation and integration of results with 
various diagnosis methods before making final conclusion on the failure.

(4) It has learning ability, which is the physical representation of diagnosis Agent having intelligence.
Diagnosis Agent can learn from current diagnosis result to enrich and supplement corresponding knowledge base, so as to improve problem-solving capacity and real-time performance of the system.

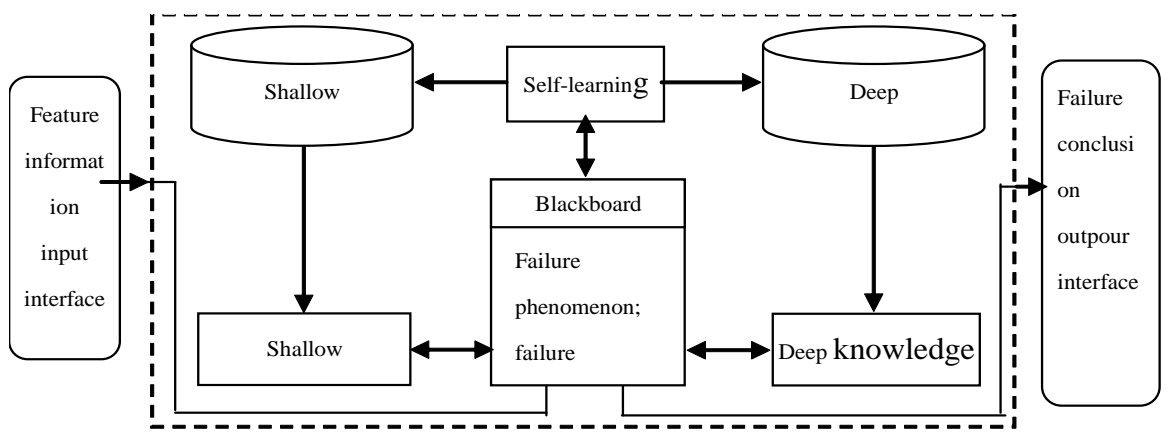

Fig. 3 Functional Configuration of Failure Diagnosis Agent

\section{Forecast Agent}

Forecast Agent functions for logical reasoning and forecast based on information on current environment and state of other Agent. Main source of information includes monitoring object, information on current state of the equipment and information on history state. The core of forecast Agent is selection of forecast model; due to singularities unavoidably exist in state information, in order to improve adaptability of forecast model, data forecast processing is necessary before selection of forecast model; forecast may only be performed after forecast model is selected, and its configuration is illustrated in Figure 4.

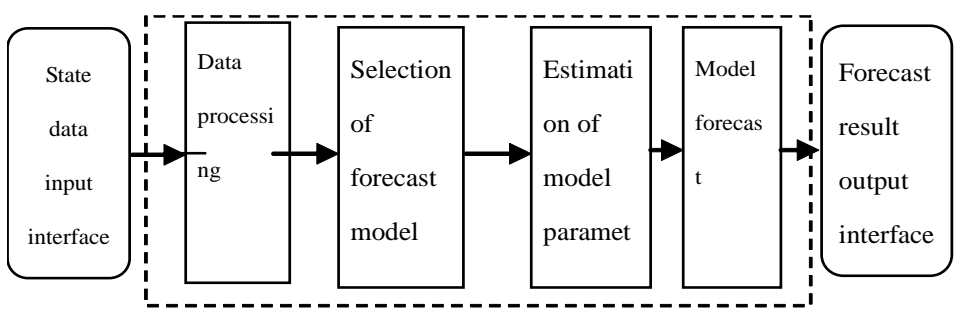

\section{E. Decision-making Agent}

Decision-making Agent mainly functions to forecast operating state tendency and failure tendency of the equipment according to current and recorded parameters about operating states and based on laws of macro-failure of such equipment with various methods, make auxiliary maintenance decision, determine maintenance time and maintenance level, so as to realize transformation from traditional regular maintenance to state-based maintenance, and its configuration is illustrated in Figure 5.

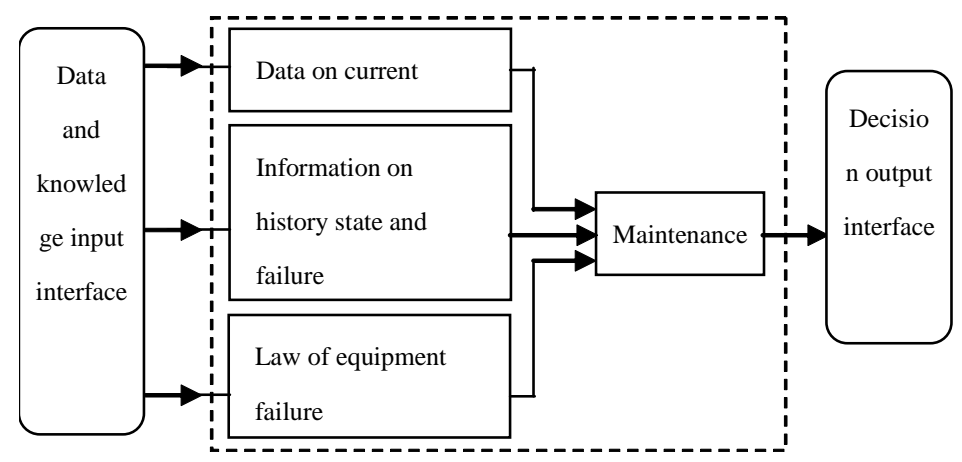

\section{F. Interfacing Agent}

Main source of information to interfacing Agent includes monitoring information and diagnosis information. Due to
Fig. 5 Functional Configuration of Decision-making Agent normal and abnormal states of diagnosis object, interfacing Agent displays and records real-time state of diagnosis object, and transmits corresponding alarm information; interfacing 
Agent serves as main channel for human-machine interaction by communication based on message mechanism, and its configuration is illustrated in Figure 6. Due to interfacing Agent mainly functions for processing related to state information, together with human interaction, it involves in complicated work, thus multithreading parallel operation is adopted to improve real-tome response capacity of the system.

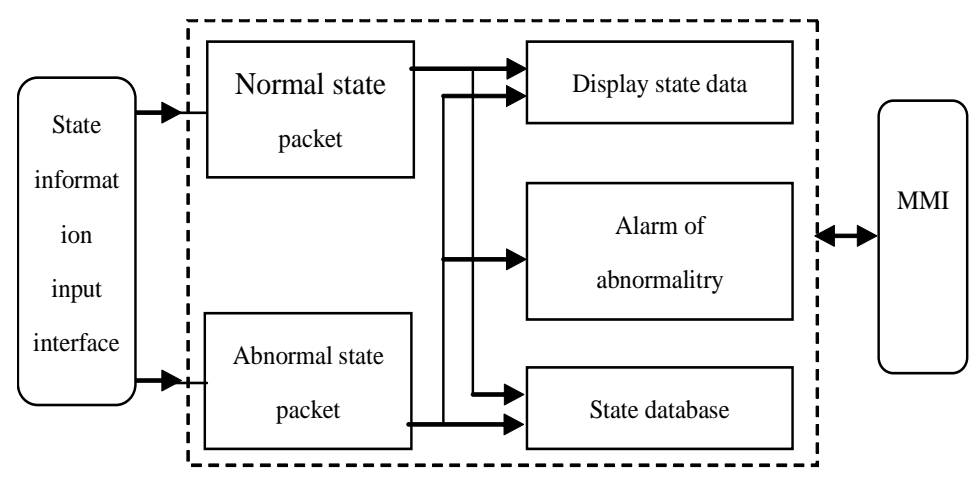

Fig. 6 Functional Configuration of Interfacing Agent

\section{G. Administration Agent}

Administration Agent mainly functions to receive monitoring and diagnosis tasks, which may be from online request for diagnosis or instructions from local user; administration Agent determines set of Agents for execution of diagnosis task according to specific task and availability of local resource, makes corresponding role distribution, and finally implements monitoring and diagnosis task. Administration Agent is the highest hierarchy of information stream in multi-Agents based diagnosis system, includes information database about the capacities, states and addresses of all Agents within the range of administration Agent, and main tasks of administration Agent are: (1) Accept and respond accordingly to local or online request for diagnosis;

(2) administration of all online communication matters, including generation of data messages, identification of network information and the like; and (3) distribution and determination of diagnosis resources.

\section{IIIConclusion}

Intelligent monitoring and diagnosis is the development trend of diagnosing failures in modern times, and monitoring and diagnosis system based on multi-Agents is a hot topic in current stage of study. This article, in the conception of modularization, unveils the way of function modeling of monitoring and diagnosis system based on multi-Agents, and expounds internal configuration of each functional Agent in the model. The model is featured by uniform information interface for "plug \& play" application, and different functional modules are available for specific monitoring object. The model is valuable to guide realizing intelligent monitoring and diagnosis system, and helpful in constructing integrated, intelligent, automated and networked diagnosis system.

\section{References}

[1]Zhu Daqi, Diagnosis of Failures of Electronic Devices: Principles \& Practice [M]. Beijing: Electronic Industry Press, 2004

[2]He Yanxiang et al, Design and Application of System Based on Agent and Multi-Agents [M]. Wuhan: Wuhan University Press, 2001
[3] Egil P. Adersen. Conceptual Modeling of Objects, a Role Modeling Approach [D]. Dr science theme Dept. of Informatics, University of Oslo. 1997

[4] Zuo Wanli, Study on Intelligent Failure Diagnosis System Based on Multi-Agents [J]. Computer and Modernization, 2003, 96 (8):4-6

[5]Zhang Jun, Zhang Rongtao, Sun Yu, Study on Modeling and Realization of Agents in Distributed Detection \& Monitoring System [J]. Computer Engineering, 2001, 27(2):93-95

[6] Wu Jinpei, Fuzzy Diagnosis Theory and Application [M]; Beijing: Science Press, 1995

[7] Gao Xinbo, Fuzzy Clustering Analysis and Application [M]; Xi'an: Xi'an University of Electronic Science and Technology Press, 2004 